H. Yokoi

Nagoya Math. J.

Vol. 33 (1968), 139-152

\title{
ON REAL QUADRATIC FIELDS CONTAINING UNITS WITH NORM -1
}

\author{
HIDEO YOKOI
}

Let $\boldsymbol{Q}$ be the rational number field, and let $K=\boldsymbol{Q}(\sqrt{D})(D>0$ a rational integer) be a real quadratic field. Then, throughout this paper, we shall understand by the fundamental unit $\varepsilon_{D}$ of $\boldsymbol{Q}(\sqrt{D})$ the normalized fundamental unit $\varepsilon_{D}>1$.

Recently $H$. Hasse investigated variously real quadratic fields with the genus 1 , but with the class number more than one ${ }^{1)}$. However, since he needed there to know a explicit form of the fundamental unit of a real quadratic field, his investigation had naturally to be restricted within the case of real quadratic fields of Richaud-Degert type whose fundamental units were already given explicitly.

In this paper, we shall give explicitly the fundamental units of real quadratic fields of the more general type than Richaud-Degert's in the case of real quadratic fields with the fundamental unit $\varepsilon$ satisfying $N \varepsilon=-1$, and consider the class number of real quadratic fields of this type as Hasse did in the case of Richaud-Degert type.

In $\$ 1$, by means of expressing any unit $\varepsilon=(t+u \sqrt{D}) / 2$ of $\boldsymbol{Q}(\sqrt{D})$ as a function of $t$, we shall give first a generating function of all real quadratic fields with the fundamental unit whose norm is equal to -1 (Theorem 1 ). In $\S 2$, by means of classifying all units $\varepsilon=(t+u \sqrt{D}) / 2$ with $N \varepsilon=-1$ by the positive value of $u$, we shall prove that in the class of $u=p$ or $2 p$ ( $p$ is 1 or prime congruent to $1 \bmod 4)$ the unit $\varepsilon=(t+u \sqrt{D}) / 2>1$ becomes the fundamental unit of $\boldsymbol{Q}(\sqrt{D})$ except for at most finite number of values of $D$ (Theorem 2 and its Corollary). Moreover, we shall show that real quadratic fields of Richaud-Degert type essentially correspond to real quadratic fields with the fundamental unit belonging to the class of $u=1$ or 2 in such classification (Proposition 2). In $\S 3$, we shall give an estima-

Received February 21, 1968.

1) Cf. H. Hasse [3]. 
tion formula from below of the class number of real quadratic fields with the fundamental unit belonging to the class of $u=p$ or $2 p$ (Theorem 3). Finally, in $\S 4$ we shall show a few examples in concrete cases of $p=5,13$.

\section{\$1. Generating function}

In order to investigate real quadratic fields with the fundamental unit whose norm is equal to -1 , we first give a generating function of those real quadratic fields. The following theorem may be already known, but since by using the theorem we can easily draw up a list of the fundamental unit $\varepsilon_{D}$ of real quadratic fields $\boldsymbol{Q}(\sqrt{D})$ satisfying $N \varepsilon_{D}=-1^{2)}$ and our investigation in this note is based on it, we dare add a simple proof of it.

Theorem 1. Let $\boldsymbol{Q}(\sqrt{D})(D>0$ square-free $)$ be a real quadratic field, then any unit $\varepsilon$ of $\boldsymbol{Q}(\sqrt{D})$ satisfying $N \varepsilon=-1$ is of the form $\varepsilon=\left(t+\sqrt{t^{2}+4}\right) / 2$ for some integer $t$, and the reverse is also true.

In particular, all real quadratic fields with the fundamental unit $\varepsilon$ satisfying $N \varepsilon=-1$ are generated by the function $\sqrt{t^{2}+4}$ over $\boldsymbol{Q}$, and conversely any field $\boldsymbol{Q}\left(\sqrt{t^{2}+4}\right)(t \neq 0)$ generated by $\sqrt{t^{2}+4}$ over $\boldsymbol{Q}$ is a real quadratic field with the fundamental unit $\varepsilon$ satisfying $N \varepsilon=-1$.

Proof. Since an unit $\varepsilon$ of a real quadratic field $\boldsymbol{Q}(\sqrt{D})(D>0$ squarefree) is an integer whose norm is equal to $\pm 1, \varepsilon$ is of the form $\varepsilon=(t+u \sqrt{D}) / 2$; $t \equiv u(\bmod 2)$, moreover $t \equiv u \equiv 0(\bmod 2)$ for the special case of $D \equiv 2,3$ $(\bmod 4)$, and $(t, u)$ satisfies Pell's equation $x^{2}-D y^{2}= \pm 4$ because of $\pm 1=N \varepsilon=\left(t^{2}-D u^{2}\right) / 4$.

Conversely, if a pair of integers $(t, u)$ satisfies Pell's equation $t^{2}-D u^{2}=-4$, then clearly $t \equiv u(\bmod 2)$ and moreover $t \equiv u \equiv 0(\bmod 2)$ for the special case of $D \equiv 2,3(\bmod 4)$. For, if we assume $t \equiv u \equiv 1(\bmod 2)$, then we have $t^{2} \equiv u^{2} \equiv 1(\bmod 4)$, and hence $t^{2}-D u^{2}=-4$ implies $D \equiv 1(\bmod 4)$. Therefore, $\varepsilon=(t+u \sqrt{D}) / 2=\left(t \pm \sqrt{D u^{2}}\right)_{1} / 2=\left(t \pm \sqrt{t^{2}+4}\right) / 2$ is a unit of $\boldsymbol{Q}(\sqrt{D})$ satisfying $N \varepsilon=-1$.

The following lemma may be partly known, but it is useful throughout this note.

Lemma 1. If Pell's equation $t^{2}-D u^{2}=-4$ is solvable for a positive squarefree integer $D$, then the prime decompositions of $D, u$ are of the following form:

\footnotetext{
2) Cf. Table 1.
} 


$$
D=2^{\delta_{1}} \prod_{i} p_{i}, \quad u=2^{\delta_{2}} \prod_{j} q_{j}^{e_{j}},
$$

where $\delta_{1}, \delta_{2}$ take the value 0 or $1, p_{i}, q_{j}$ are congruent to $1 \bmod 4$, and $e_{j}$ are positive integers. Moreover, $D \equiv 2(\bmod 4)$ implies $t \equiv 0(\bmod 2)$, which is equivalent to $u \equiv 0(\bmod 2)$.

Proof. If Pell's equation $t^{2}-D u^{2}=-4$ is solvable, then $t^{2} \equiv-4(\bmod$ $\left.D u^{2}\right)$ holds, and hence for any odd prime factor $p$ of $D u^{2}$, we have $t^{2} \equiv-4$ $(\bmod p)$. Therefore, we get $1=\left(\frac{-4}{p}\right)=(-1)^{\frac{p-1}{2}}$, which implies $p \equiv 1$ $(\bmod 4)$.

Next, if $u \equiv 0(\bmod 4)$ holds, then $t^{2}-D u^{2}=-4$ implies $t \equiv 0(\bmod 2)$, and hence we may put $u=4 u_{0}, t=2 t_{0}$, and we have $t_{0}^{2}-4 D u_{0}^{2}=-1$. Therefore, we get $t_{0}^{2} \equiv-1(\bmod 4)$, which is a contradiction. The remaining part is clear from $t^{2}-D u^{2}=-4$.

\section{§2. Fundamental unit}

We first give the fundamental unit of real quadratic fields of two types.

Proposition 1. (i) If $D=t^{2}+4(t>0)$ is square-free, then $\varepsilon_{D}=\left(t+\sqrt{t^{2}+4}\right) / 2$ is the fundamental unit of the real quadratic field $\boldsymbol{Q}(\sqrt{D})$ and $N \varepsilon_{D}=-1$.

(ii) If $D=t_{0}^{2}+1\left(0<t_{0} \neq 2\right)$ is square-free, then $\varepsilon_{D}=t_{0}+\sqrt{t_{0}^{2}+1}$ is the fundamental unit of the real quadratic field $\boldsymbol{Q}(\sqrt{D})$ and $N \varepsilon_{D}=-1$.

Proof. Let $(x, y)=(t, u)$ be the least positive integral solution of Pell's equation $x^{2}-D y^{2}=-4$ (if exists), then $\varepsilon_{D}=(t+u \sqrt{D}) / 2$ is the fundamental unit of the real quadratic field $\boldsymbol{Q}(\sqrt{D})$ and $N \varepsilon_{D}=-1$. Therefore, in the special case of $y=u=1$, i.e. $t^{2}-D=-4, \quad \varepsilon_{D}=(t+u \sqrt{D}) / 2=\left(t+\sqrt{t^{2}+4}\right) / 2$ is certainly the fundamental unit of $\mathbf{Q}\left(\sqrt{t^{2}+4}\right)$ provided that $D=t^{2}+4$ is square-free. In the case of $y=u=2$, we get $t \equiv 0(\bmod 2)$ from lemma 1 , and hence we may put $t=2 t_{0}$, and $t_{0}^{2}-D=-1$ holds. Hence, $\varepsilon_{D}=(t+u \sqrt{D}) / 2=t_{0}+\sqrt{t_{0}^{2}+1}$ is the fundamental unit of $\boldsymbol{Q}\left(\sqrt{t_{0}^{2}+1}\right)$ provided that $D=t_{0}^{2}+1$ is square-free and $D$ is not of the above mentioned type (i). However, $D=t_{0}^{2}+1=t^{2}+4$ holds for some integers $t_{0}, t$ if and only if $t_{0}$ is equal to 2, i.e. $D=5=2^{2}+1=1^{2}+4$. Thus, the proposition 1 is proved in both cases. 
Probably, the following result of Richaud-Degert ${ }^{3}$ is only one that gives explicitly the fundamental unit of real quadratic fields of certain type.

Lemma 2 (Richaud-Degert). Let $\boldsymbol{Q}(\sqrt{D})(D>0$ square-free) be a real quadratic field, and put $D=n^{2}+r(-n<r \leqq n)$. Then, if $4 n \equiv 0 \quad(\bmod r)$ holds, the fundamental unit $\varepsilon_{D}$ of $\boldsymbol{Q}(\sqrt{D})$ is of the following form:

$$
\begin{aligned}
& \varepsilon_{D}=n+\sqrt{D} \text { with } N \varepsilon_{D}=-\operatorname{sgn} r \text { for }|r|=1, \\
&\text { (except for } D=5, n=2, r=1), \\
& \varepsilon_{D}=(n+\sqrt{D}) / 2 \text { with } N \varepsilon_{D}=-\operatorname{sgn} r \text { for }|r|=4, \\
& \varepsilon_{D}=\left[\left(2 n^{2}+r\right)+2 n \sqrt{D}\right] / r \text { with } N \varepsilon_{D}=1 \text { for }|r| \neq 1,4 .
\end{aligned}
$$

Such type of real quadratic fields that the assumption of this lemma is satisfied we shall call simply R-D type. Then the following proposition shows a relation between the type of real quadratic fields in proposition 1 and R-D type in the case of real quadratic fields with the fundamental unit whose norm is equal to -1 .

Proposition 2. A real quadratic field $\boldsymbol{Q}(\sqrt{D})(D>0$ square-free) with the fundamental unit whose norm is equal to -1 is of $R-D$ type if and only if $D$ is of the form $D=t^{2}+4$ or $t_{0}^{2}+1\left(t, t_{0}>0\right.$ integer $)$ except for $D=5,13$; in other words, if and only if $u$ in the least positive integral solution $(x, y)=(t, u)$ of Pell's equation $x^{2}-D y^{2}=-4$ is equal to 1 or 2 .

Proof. Let $\boldsymbol{Q}(\sqrt{D})(D>0$ square-free) be a real quadratic field with the fundamental unit whose norm is equal to -1 . Then, if $\boldsymbol{Q}(\sqrt{D})$ is of R-D type, $D$ is of the form $D=t^{2}+4$ or $t_{0}^{2}+1,\left(t, t_{0}>0\right.$ integers) by lemma 2 , and hence it follows from proposition 1 that in the least positive integral solution $(x, y)=(t, u)$ of Pell's equation $x^{2}-D y^{2}=-4$ is equal to 1 or 2 .

Conversely, if $u=2$, i.e. $D=t_{0}^{2}+1$, then $Q(\sqrt{D})$ is clearly of R-D type. On the other hand, in the case of $u=1$, i.e. $D=t^{2}+4, \boldsymbol{Q}(\sqrt{D})$ is of R-D type if and only if $t \geqq 4$ holds. However, in the case of $t=2, D$ is equal to 8 and is not square-free.

Therefore, except for $D=5$ with $t=1$ and $D=13$ with $t=3$, it is equivalent to $u=1$ or 2 that the real quadratic field $\boldsymbol{Q}(\sqrt{D})$ with the fundamental unit whose norm is equal to -1 is of R-D type.

3) Cf. G. Degert [2] and C. Richaud [6]. 
Thus, both $\boldsymbol{Q}(\sqrt{5})$ and $\boldsymbol{Q}(\sqrt{13})$ are not of R-D type, but both values of $u$ in the least positive integral solution $(x, y)=(t, u)$ of Pell's equation $x^{2}-D y^{2}=-4$ are equal to 1 . Hence, from now, we shall understand R-D type in such a wide sense that it contains both $\boldsymbol{Q}(\sqrt{5})$ and $\boldsymbol{Q}(\sqrt{13})$.

In order to give explicitly the fundamental unit of real quadratic fields of a new type different from R-D's, we must prepare the following three lemmas:

Lemma 3. For any prime $p$ satisfying $p \equiv 1(\bmod 4)$, an unit $\varepsilon$ of a real quadratic field $\boldsymbol{Q}(\sqrt{D})$ that is of the form $(t+p \sqrt{D}) / 2$ or $t+p \sqrt{D}(D>0$ squarefree) and that satisfies $N \varepsilon=-1$ is the fundamental unit of $\boldsymbol{Q}(\sqrt{D})$ if and only if the real quadratic field $\boldsymbol{Q} \sqrt{D}$ ) is not of $R-D$ type.

Proof. Let $\varepsilon_{0}=\left(t_{0}+u_{0} \sqrt{D}\right) / 2(D>0$ square-free $)$ be the fundamental unit of the real quadratic field $\boldsymbol{Q}(\sqrt{D})$, then the norm of $\varepsilon_{0}$ is equal to -1 and there exists an odd integer $n$ satisfying $\varepsilon=\varepsilon_{0}^{n}$. If we put for this odd integer $n 2^{n} \varepsilon_{0}^{n}=\left(t_{0}+u_{0} \sqrt{D}\right)^{n}=T+U \sqrt{D}$, then we have $U={ }_{n} C_{1} t_{0}^{n-1} u_{0}+{ }_{n} C_{3} t_{0}^{n-3} u_{0}^{3} D+$ $\cdots+{ }_{n} C_{n-2} t_{0}^{2} u_{0}^{n-2} D^{\frac{n-3}{2}}+{ }_{n} C_{n} u_{0}^{n} D^{\frac{n-1}{2}} \equiv 0\left(\bmod u_{0}\right)$, while we have $U=2^{n-1} p$ or $2^{n} p$. Hence, in the case of $u_{0} \equiv 1(\bmod 4)$, we get $p \equiv 0\left(\bmod u_{0}\right)$, which implies $u_{0} \equiv 1$ or $p$. In the case of $u_{0} \equiv 1(\bmod 4)$, we may put by lemma $1 u_{0}=2 u_{0}^{\prime}, \quad u_{0}^{\prime} \equiv 1(\bmod 4)$. Hence, we get $p \equiv 0\left(\bmod u_{0}^{\prime}\right)$, which implies $u_{0}^{\prime}=1$ or $p$. Therefore, the condition $u_{0}=p$ or $2 p$ is equivalent to $u_{0} \neq 1,2$. On the other hand, since the condition $\varepsilon_{0}=\varepsilon$ is equivalent to $u_{0}=p$ or $2 p$, it follows from proposition 2 that $\varepsilon=\varepsilon_{0}$ holds if and only if the real quadratic field $\boldsymbol{Q}(\sqrt{D})$ is not of R-D type.

Lemma 4. For any prime $p$ satisfying $p \equiv 1(\bmod 4)$, there are two uniquely determined integers $a, b$ such that $a^{2}+4=b p^{2}, 0<a<p^{2}$. Moreover, for these $p, a, b, D=p^{2} m^{2} \pm 2 a m+b(m>0)$ is congruent to 1 mod 4 or congruent to 4 or $8 \bmod 16$, and Pell's equation $t^{2}-D u^{2}=-4$ is always solvable. $\left.{ }^{4}\right)$

Proof. Since for any prime $p$ congruent to $1 \bmod 4$ we get $\left(\frac{-4}{p}\right)=1$, congruence $x^{2} \equiv-4(\bmod p)$ is solvable, and hence congruence $x^{2} \equiv-4$ $\left(\bmod p^{2}\right)$ is also solvable. Among the solutions of this congruence $x^{2} \equiv-4$

4) L. Rédei notes in [5] that if Pell's equation $t^{2}-d u^{2}=-1$ is solvable for some integer $d=d_{0}$, then the Pell's equation is also solvable for $d=u_{0}^{2} m^{2}+2 t_{0} m+d_{0}$, where $\left(t_{0}, u_{0}\right)$ is any positive integral solution of $t^{2}-d_{0} u^{2}=-1$ and $m$ is any integer. 
$\left(\bmod p^{2}\right)$, there exists only one solution $x \equiv \pm a\left(\bmod p^{2}\right)$ satisfying $0<a<p^{2}$. For this positive integer $a$, there is a unique integer $b$ satisfying $a^{2}+4=b p^{2}$. Conversely, if $a^{2}+4=b p^{2}$ holds, then $x \equiv \pm a\left(\bmod p^{2}\right)$ is a solution of congruence $x^{2} \equiv-4\left(\bmod p^{2}\right)$.

Next, set $D=p^{2} m^{2} \pm 2 a m+b, \quad t=p^{2} m \pm a, \quad u=p \quad(m>0)$, then Pell's equation $t^{2}-D u^{2}=-4$ is certainly satisfied by these $D, t, u$. Therefore, if we note only that $p^{2} \equiv 1(\bmod 4)$ and $t^{2}+4=D p^{2}$, it is easy to see that $D \equiv 1(\bmod 4)$ for odd $t$, and $D \equiv 0(\bmod 4)$ for even $t$. In the case of $D \equiv 0(\bmod 4)$, we may put $D=4 D_{0}, t=2 t_{0}$, and get $t_{0}^{2}+1=D_{0} p^{2}$. Hence, we obtain similarly $D_{0} \equiv 2(\bmod 4)$ for odd $t_{0}$ and $D_{0} \equiv 1(\bmod 4)$ for even $t$. Thus, we have $D=4 D_{0} \equiv 4$ or $8(\bmod 16)$.

In order to prove theorem 2 we require another lemma, which is itself of some interest.

Lemma 5. For any integers $a>0, b, c$ satisfying $b \neq 0(\bmod a)$, there exist at most a finite number of such natural $n$ that $f(n)=a^{2} n^{2}+b n+c$ is square.

Proof. It follows from the assumption $b \neq \equiv 0(\bmod a)$ that an integer $k$ satisfying $\left|\frac{b}{2 a}-k\right|<\frac{1}{2}$ is uniquely determined. By using this integer $k$, we rewrite $f(n)$ in the following form:

$$
f(n)=a^{2} n^{2}+b n+c=(a n+k)^{2}+(b-2 a k) n+\left(c-k^{2}\right) .
$$

Then, since $|b-2 a k|<a$, the inequality

$$
-(a n+k)<(b-2 a k) n+\left(c-k^{2}\right)<a n+k
$$

holds for all natural $n$ except at most finite number of cases. Moreover, since $b-2 a k \neq 0$, we know that

$$
(b-2 a k) n+\left(c-k^{2}\right) \neq 0
$$

holds for all natural $n$ except for at most one.

On the other hand, the above inequality shows that $(b-2 a k) n+\left(c-k^{2}\right)$ is the nearest integer to $\sqrt{f(n)}$ in absolute value. Therefore, $f(n)=a^{2} n^{2}+b n+c$ does not become square for any natural $n$ apart from a finite number of exceptions. The lemma is thus proved.

Theorem 2. For any prime $p$ congruent to $1 \bmod 4$, let, $a, b$ denote the integer in lemma 4 satisfying $a^{2}+4=b p^{2}\left(0<a<p^{2}\right)$. Then, there exists an integer $D_{0}=D_{0}(p)$ such that if $D=p^{2} m^{2} \pm 2 a m+b(m \geqq 0)$ has no square factor 
except 4 , and if $D \geqq D_{0}$, the real quadratic field $\boldsymbol{Q}(\sqrt{D})$ is not of $R-D$ type. Therefore, the fundamental unit $\varepsilon_{D}$ of $\boldsymbol{Q}(\sqrt{D})$ is of the following form:

$$
\varepsilon_{D}=\left\{\begin{array}{l}
{\left[\left(p^{2} m \pm a\right)+p \sqrt{D}\right] / 2 \cdots \cdots \cdot \text { square-free }} \\
\left(p^{2} m \pm a\right) / 2+p \sqrt{D / 4} \cdots \cdots \text { otherwise }
\end{array}\right.
$$

and $N \varepsilon_{D}=-1$.

Proof. Since Pell's equation $t^{2}-D u^{2}=-4$ is satisfied by $D=p^{2} m^{2} \pm 2 a m+b$, $t=p^{2} m \pm a, u=p, \varepsilon=\left[\left(p^{2} m \pm a\right)+p \sqrt{D}\right] / 2$ is an unit of the real quadratic field $\boldsymbol{Q}(\sqrt{D})$, and $N \varepsilon=-1$. Moreover, by our assumptions $a^{2}+4=b p^{2}$ and $p \equiv 1(\bmod 4)$ we have $2 a \equiv 0(\bmod p)$. Therefore, in the case that $D$ is square-free, it follows from lemma 5 that both $D-1=p^{2} m^{2} \pm 2 a m+b-1$ and $D-4=p^{2} m^{2} \pm 2 a m+b-4$ are never square for any natural $m$ except at most a finite number, and hence by lemma 2 the quadratic field $\boldsymbol{Q}(\sqrt{D})$ is not of $R-D$ type for any natural $m$ except at most a finite number. In the case of $D=4 D_{0}\left(D_{0}>0\right.$ square-free), we have $t=p^{2} m \pm a \equiv 0(\bmod 2)$ by lemma 1 , and hence $m \equiv a(\bmod 2)$. By our assumptions $a^{2}+4=b p^{2}$, $p \equiv 1(\bmod 4), a \equiv 0(\bmod 2)$ is equivalent to $b \equiv 0(\bmod 4)$, and $a \equiv 1$ $(\bmod 2)$ is equivalent to $b \equiv 1(\bmod 4)$.

Therefore, in the case of $m \equiv 0(\bmod 2)$, we may put $m=2 m_{0}, b=4 b_{0}$ and get $D_{0}=D / 4=p^{2} m_{0}^{2} \pm a m_{0}+b_{0}$. Since $a \not \equiv 0(\bmod p)$, it follows from lemma 5 that both $D_{0}-1$ and $D_{0}-4$ are never square for any natural $m$ except at most a finite number. In the case of $m \equiv 1(\bmod 2)$, we may put $m=2 m_{0}+1, \quad b=4 b_{0}+1$ and get $D_{0}=D / 4=p^{2} m_{0}^{2}+\left(p^{2} \pm a\right) m_{0}+\left(b_{0}+\left(p^{2}\right.\right.$ $+1 \pm 2 a) / 4)$. Since $p^{2} \pm a \equiv \pm a \neq \equiv 0(\bmod p)$, it follows from lemma 5 that both $D_{0}-1$ and $D_{0}-4$ are never square for any natural $m_{0}$ except at most a finite number. Thus, for both types of $m$, we see at once from lemma 2 that the quadratic field $\boldsymbol{Q}(\sqrt{D})=\boldsymbol{Q}(\sqrt{D} / 4)$ is never of $R-D$ type for any natural $m$ up to at most a finite number of exceptions.

Therefore, it was proved by lemma 3 for both types of $D$ that there exists an integer $D_{0}=D_{0}(p)$ such that the above mentioned unit $\varepsilon=\left[\left(m p^{2} \pm a\right)\right.$ $+p \sqrt{D}] / 2$ is the fundamental unit of $\boldsymbol{Q}(\sqrt{D})$ provided that $D$ has no square factor except 4 , and that $D \geqq D_{0}(p)$.

This theorem implies the following sufficient condition for an unit $\varepsilon$ of a real quadratic field $\boldsymbol{Q}(\sqrt{D})(D>0$ square-free) satisfying $N \varepsilon=-1$ to be the fundamental unit. 
Corollary. For any prime $p$ congruent to $1 \bmod 4$, there exists an integer $D_{0}=D_{0}(p)$ such that if for some square-free $D$ satisfying $D \geqq D_{0}$ the real quadratic field $\boldsymbol{Q}(\sqrt{D})$ contains an unit $\varepsilon$ of the form $\varepsilon=\left(t_{0}+p_{\sqrt{D}}\right) / 2$ or $t_{0}+p_{\sqrt{D}}$ and $N \varepsilon=-1$ holds, then the unit $\varepsilon$ is the fundamental unit of $\boldsymbol{Q}(\sqrt{D})$.

Proof. In the case of $\varepsilon=\left(t_{0}+p \sqrt{D}\right) / 2,-1=N \varepsilon=\left(t_{0}^{2}-D p^{2}\right) / 4$ implies $t_{0}^{2}+4=D p^{2}$. Hence, $x \equiv t_{0}\left(\bmod p^{2}\right)$ is a solution of $x^{2} \equiv-4\left(\bmod p^{2}\right)$. On the other hand, let $a, b$ be as in lemma 4 satisfying $a^{2}+4=b p^{2}$, then we get $t_{0}=p^{2} m_{1} \pm a$ for some integer $m_{1} \geqq 0$. Therefore, $D p^{2}=t_{0}^{2}+4=\left(p^{2} m_{1} \pm a\right)^{2}$ $+4=p^{2}\left(p^{2} m_{1}^{2} \pm 2 a m_{1}+b\right)$ implies $D=p^{2} m_{1}^{2} \pm 2 a m_{1}+b\left(m_{1} \geqq 0\right)$. If we choose $D_{0}$ in theorem 2 as $D_{0}=D_{0}(p)$ in question, and consider square-free $D$ satisfying $D \geqq D_{0}$, then it follows from theorem 2 that the unit $\varepsilon=\left(t_{0}+p \sqrt{D}\right) / 2$ is the fundamental unit of $\boldsymbol{Q}(\sqrt{D})$.

In the case of $\varepsilon=t_{0}+p \sqrt{D},-1=t_{0}^{2}-D p^{2}$ implies $t_{0}^{2}+1=D p^{2}$. Hence, there exists an integer $m_{2} \geqq 0$ satisfying $2 t_{0}=p^{2} m_{2} \pm a$, because $x \equiv 2 t_{0}$ $\left(\bmod p^{2}\right)$ is a solution of $x^{2} \equiv-4\left(\bmod p^{2}\right)$. Therefore, $(4 D) p^{2}=\left(2 t_{0}\right)^{2}+4$ $=\left(p^{2} m_{2} \pm a\right)^{2}+4=p^{2}\left(p^{2} m_{2}^{2} \pm 2 a m_{2}+b\right)$ implies $4 D=p^{2} m_{2}^{2} \pm 2 a m_{2}+b\left(m_{2} \geqq 0\right)$. If we choose $D_{0}$ in theorem 2 as $D_{0}=D_{0}(p)$ in question and consider squarefree $D$ satisfying $D_{0} \leqq 4 D$, it follows from theorem 2 that the unit $\varepsilon=t_{0}+p_{\sqrt{D}} \bar{D}$ is the fundamental unit of $\boldsymbol{Q}(\sqrt{D})$. Thus, in both cases the corollary is proved.

\section{§3. Class number}

In this §, we give an estimation formula from below of the class number of those real quadratic fields whose fundamental unit was given in $\$ 2$. To this purpose we require the following lemma of Davenport-Ankeny-Hasse:

Lemma 6. (Davenport-Ankeny-Hasse $\left.)^{5}\right)$ Let $\boldsymbol{Q}(\sqrt{D})(D>0$ square-free $)$ be a real quadratic field with the fundamental unit $\varepsilon_{D}=(t+u \sqrt{D}) / 2(t, u>0)$. Then, if Pell's equation $\left(x^{2}-D u^{2}\right) / 4= \pm m$ ( $m$ not square) is solvable, the following inequality holds:

$$
\left\{\begin{array}{l}
m \geqq(t-2) / u^{2} \quad \text { for } \quad N \varepsilon_{D}=1, \\
m \geqq t / u^{2} \quad \text { for } \quad N \varepsilon_{D}=-1 .
\end{array}\right.
$$

5) Cf. N.C. Ankeny, S. Chowla and H. Hasse [1] and H. Hasse [3]. 
Let us quote this boundary $s=t / u^{2}$ for $N \varepsilon_{D}=-1$ in lemma 6 as Hasse's boundary (in the lemma of D-A-H).

Theorem 3. For any prime $p$ congruent to $1 \bmod 4$, let $a, b$ denote the integers in lemma 4 satisfying $a^{2}+4=b p^{2}\left(0<a<p^{2}\right)$, and let $D_{0}=D_{0}(p)$ be the integer in theorem 2. Furthermore, set $D=p^{2} m^{2} \pm 2 a m+b$ for any integer $m$ bigger than $4 p$, and consider $D$ bigger than $D_{0}(p)$. Then, if $D$ has no square factor except 4 and $p$ splits in the real quadratic field $\boldsymbol{Q}(\sqrt{D})$ into two conjugate prime ideals with the degree one, these prime ideals are not principal. Therefore, the class number $h$ of $\boldsymbol{Q}(\sqrt{D})$ is bigger than one and the following estimation from below holds:

$$
\begin{aligned}
& h \geqq \frac{\log \sqrt{D p^{2}-4}}{\log p}-2 \quad \text { for } D \equiv 1 \quad(\bmod 2), \\
& h \geqq \frac{\log \frac{1}{4} \sqrt{D p^{2}-4}}{\log p}-2 \text { for } D \equiv 0 \quad(\bmod 2) .
\end{aligned}
$$

Proof. In the case of $D \equiv 1(\bmod 2), D$ is square-free from the assumption, and hence by theorem 2 the fundamental unit of $\boldsymbol{Q}(\sqrt{D})$ is $\varepsilon_{D}=\left[\left(m p^{2}\right.\right.$ $\pm a)+p \sqrt{D}] / 2$ provided $D \geqq D_{0}(p)$. Therefore, it follows from lemma 6 that Hasse's boundary is $s=\left(m p^{2} \pm a\right) / p^{2}=m \pm a / p^{2}\left(0<a / p^{2}<1\right)$. In the case of $D \equiv 0(\bmod 2)$, we have $D \equiv 0(\bmod 4)$ by lemma 4 , and $D_{0}=4 / D$ is squarefree. Therefore, by theorem 2 the fundamental unit of $\boldsymbol{Q}(\sqrt{D})$ is $\varepsilon_{D}=$ $\left(m p^{2} \pm a\right) / 2+p \sqrt{D / 4}$ provided $D \geqq D_{0}(p)$, and hence by lemma 6 Hasse's boundary is $s=\left(m p^{2} \pm a\right) / 4 p^{2}=m / 4 \pm a / 4 p^{2}\left(0<a / 4 p^{2}<1 / 4\right)$. For any integer $m$ bigger than $p$ (in the first case) or $4 p$ (in the second case), the prime $p$ is smaller than Hasse's boundary $s$ i.e. $p<s$.

If we assume that the prime $p$ splits into two conjugate principal ideals $\mathfrak{p}, \mathfrak{p}^{\prime}$ with the degree one in $\boldsymbol{Q}(\sqrt{D})$, then Pell's equation $\left(x^{2}-D y^{2}\right) / 4= \pm p$ is solvable, and hence lemma 6 implies $p>s$, which is contrary to the above assertion $p<s$. Therefore, if the prime $p$ splits into two conjugate prime ideals $\mathfrak{p}, \mathfrak{p}^{\prime}$ with the degree one in $\boldsymbol{Q}(\sqrt{D})$, then the prime $\mathfrak{p}, \mathfrak{p}^{\prime}$ are not principal. Moreover, the order of those prime ideals $\mathfrak{p}, \mathfrak{p}^{\prime}$ in the ideal class group of $\boldsymbol{Q}(\sqrt{D})$ is bigger than one and it is a factor of the ideal class number $h$ of $\boldsymbol{Q}(\sqrt{D})$. Hence, in the case of $D \equiv 1(\bmod 2)$, we have

$$
p^{h} \geqq s=\frac{m p^{2} \pm a}{p^{2}}=\frac{\sqrt{D p^{2}-4}}{p^{2}},
$$


which implies

$$
h \geqq \frac{\log \sqrt{D p^{2}-4}}{\log p}-2,
$$

and similarly in the case of $D \equiv 0(\bmod 2)$, we have

$$
p^{h} \geqq s=\frac{m p^{2} \pm a}{4 p^{2}}=\frac{\sqrt{D p^{2}-4}}{4 p^{2}},
$$

which implies

$$
h \geqq \frac{\log \frac{1}{4} \sqrt{D p^{2}-4}}{\log p}-2 .
$$

Thus, the theorem is completely proved.

Remark 1. In the case of $D \pm D_{0}(p), \quad \varepsilon=\left[\left(m p^{2} \pm a\right) / 2+p \sqrt{D}\right]$ and $\varepsilon=\left(m p^{2} \pm a\right) / 2+p \sqrt{D / 4}$ are not always the fundamental unit of the real quadratic field $\boldsymbol{Q}(\sqrt{D})$, but they are always an unit of $\boldsymbol{Q}(\sqrt{D})$ satisfying $N \varepsilon=-1$. On the other hand, it is not always necessary in lemma 6 that the unit $\varepsilon$ is the fundamental unit of $\boldsymbol{Q}(\sqrt{D})$; it is sufficient that $\varepsilon$ is an unit, as we can see easily from proof of lemma 6 . Therefore, we can remove the condition $D \geqq D_{0}(p)$ in theorem 3 .

Remark 2. In the case of real quadratic fields of $R-D$ type, H. Hasse obtained already in [3] an explicit estimation formula as in theorem 3, and in the case of $\boldsymbol{Q}\left(\sqrt{a^{2}+1}\right)$ T. Nagell also treated in [4] a similar problem.

\section{\$4. Examples}

[I] The case of $p=5$.

$$
\begin{aligned}
& a=11, \quad b=5, \quad D_{0}(p)=61, \\
& t=25 m \pm 11, \quad D=25 m^{2} \pm 22 m+5 .
\end{aligned}
$$

(1) If $m \equiv 0(\bmod 2)$, then $D \equiv 1(\bmod 4)$, and hence the fundamental unit is

$$
\varepsilon=\left[(25 m \pm 11)+5 \sqrt{25 m^{2} \pm 22 m+5}\right] / 2 .
$$

Hasse's boundary is $s=m \pm 11 / 25$.

Hence $s>5 \Leftrightarrow m \geqq 6$.

(2) If $m \equiv 1(\bmod 2)$, then $D \equiv 0(\bmod 4)$, and hence the fundamental unit is 


$$
\varepsilon=(25 m \pm 11) / 2+5 \sqrt{\left(25 m^{2} \pm 22 m+5\right) / 4},
$$

Hasse's boundary is $s=m / 4 \pm 11 / 100$.

Hence $s>5 \Leftrightarrow m \geqq 21$.

$D_{0}=D / 4 \equiv 2(\bmod 4) \Leftrightarrow m \equiv 1(\bmod 4)$,

$D_{0}=D / 4 \equiv 1(\bmod 4) \Leftrightarrow m \equiv-1(\bmod 4)$.

[II] The case of $p=13$.

$a=29, \quad b=5, \quad D_{0}(p)=58$,

$t=169 m \pm 29, \quad D=169 m^{2} \pm 58 m+5$.

(1) If $m \equiv 0(\bmod 2)$, then $D \equiv 1(\bmod 4)$, and hence the fundamental unit is

$$
\varepsilon=\left[(199 m \pm 29)+13 \sqrt{169 m^{2} \pm 58 m+5}\right] / 2,
$$

Hasse's boundary is $s=m \pm 29 / 169$.

Hence $s>13 \Leftrightarrow m \geqq 14$.

(2) If $m \equiv 1(\bmod 2)$, then $D \equiv 0(\bmod 4)$, and hence the fundamental unit is

$$
\varepsilon=(169 m \pm 29) / 2+13 \sqrt{\left(169 m^{2} \pm 58 m+5\right) / 4},
$$

Hasse's boundary is $s=m / 4 \pm 29 / 676$.

Hence $s>13 \Leftrightarrow m \geqq 53$

$D_{0}=D / 4 \equiv 2(\bmod 4) \Leftrightarrow m \equiv 1(\bmod 4)$,

$D_{0}=D / 4 \equiv 1(\bmod 4) \Leftrightarrow m \equiv-1(\bmod 4)$.

\section{REFERENCES}

[1] N.C. Ankeny, S. Chowla and H. Hasse, On the class number of the real subfield of a cyclotomic field. J. reine angew. Math. 217 (1965), 217-220.

[2] G. Degert, Über die Bestimmung der Grundeinheit gewisser reell-quadratischer Zahlkörper. Abh. math. Sem. Univ. Hamburg 22 (1958), 92-97.

[ 3 ] H. Hasse, Über mehrklassige, aber eingeschlechtige reell-quadratische Zahlkörper. Elemente der Mathematik 20 (1965), 49-59.

[4] T. Nagell, Bemerkung über die Klassenzahl reell-quadratischer Zahlkörper. Det Kongelige Norske Videnskabens Selskab, Forhandlinger 11 (1938), 7-10.

[ 5 ] L. Rédei, Über die Pellsche Gleichung $t^{2}-d u^{2}=-1$. J. reine angew. Math. 173 (1935), 193-221.

[6] C. Richaud, Sur la résolution des équations $x^{2}-A y^{2}= \pm 1$. Atti Accad. pontif. Nuovi Lincei (1866), 177-182. 
Table 1

$\varepsilon_{D}=(t+u \sqrt{D}) / 2$

\begin{tabular}{|c|c|c|c|c|c|c|c|}
\hline$t$ & $D$ & $u$ & & $t$ & $D$ & $u$ & \\
\hline 1 & 5 & 1 & & 31 & $965=5 \cdot 193$ & 1 & \\
\hline 2 & 2 & 2 & & 32 & 257 & 2 & \\
\hline 3 & 13 & 1 & & 33 & 1093 & 1 & \\
\hline 4 & 5 & 2 & $\varepsilon_{5}^{36)}$ & 34 & $290=2 \cdot 5 \cdot 29$ & 2 & \\
\hline 5 & 29 & 1 & & 35 & 1229 & 1 & \\
\hline 6 & $10=2 \cdot 5$ & 2 & & 36 & 13 & 10 & $\varepsilon_{13}^{3}$ \\
\hline 7 & 53 & 1 & & 37 & 1373 & 1 & \\
\hline 8 & 17 & 2 & & 38 & $362=2 \cdot 181$ & 2 & \\
\hline 9 & $85=5 \cdot 17$ & 1 & & 39 & 61 & 5 & \\
\hline 10 & $26=2 \cdot 13$ & 2 & & 40 & 401 & 2 & \\
\hline 11 & 5 & 5 & $\varepsilon_{5}^{5}$ & 41 & $1685=5 \cdot 337$ & 1 & \\
\hline 12 & 37 & 2 & & 42 & $442=2 \cdot 13 \cdot 17$ & 2 & \\
\hline 13 & 173 & 1 & & 43 & $1853=17 \cdot 109$ & 1 & \\
\hline 14 & 2 & 10 & $\varepsilon_{2}^{3}$ & 44 & $485=5 \cdot 97$ & 2 & \\
\hline 15 & 229 & 1 & & 45 & 2029 & 1 & \\
\hline 16 & $65=5 \cdot 13$ & 2 & & 46 & $530=2 \cdot 5 \cdot 53$ & 2 & \\
\hline 17 & 293 & 1 & & 47 & 2213 & 1 & \\
\hline 18 & $82=2 \cdot 41$ & 2 & & 48 & 577 & 2 & \\
\hline 19 & $365=5 \cdot 73$ & 1 & & 49 & $2405=5 \cdot 13 \cdot 37$ & 1 & \\
\hline 20 & 101 & 2 & & 50 & $626=2 \cdot 313$ & 2 & \\
\hline 21 & $445=5 \cdot 89$ & 1 & & 51 & $2605=5 \cdot 521$ & 1 & \\
\hline 22 & $122=2 \cdot 61$ & 2 & & 52 & 677 & 2 & \\
\hline 23 & $533=13 \cdot 41$ & 1 & & 53 & $2813=29 \cdot 97$ & 1 & \\
\hline 24 & $145=5 \cdot 29$ & 2 & & 54 & $730=2 \cdot 5 \cdot 73$ & 2 & \\
\hline 25 & $629=17 \cdot 37$ & 1 & & 55 & $3029=13 \cdot 233$ & 1 & \\
\hline 26 & $170=2 \cdot 5 \cdot 17$ & 2 & & 56 & $785=5.157$ & 2 & \\
\hline 27 & 733 & 1 & & 57 & 3253 & 1 & \\
\hline 28 & 197 & 2 & & 58 & $842=2 \cdot 421$ & 2 & \\
\hline 29 & 5 & 13 & $\varepsilon_{5}^{7}$ & 59 & $3485=5 \cdot 17 \cdot 41$ & 1 & \\
\hline 30 & $226=2 \cdot 113$ & 2 & & 60 & $901=17 \cdot 53$ & 2 & \\
\hline
\end{tabular}

6) $\varepsilon_{5}^{3}=(4+2 \sqrt{5}) / 2$ means the third power of the fundamental unit $\varepsilon_{5}$ of the real quadratic field $Q(\sqrt{5})$, and etc. 
Table 2

The case of $p=5$.

\begin{tabular}{|c|c|c|c|c|c|c|}
\hline$t$ & $D$ & $u$ & $m$ & $t$ & $D$ & $u$ \\
\hline & & & 0 & 11 & $5 ; \varepsilon_{5}^{5}$ & 5 \\
\hline 14 & $2 ; \varepsilon_{2}^{3}$ & 10 & 1 & 36 & $13 ; \varepsilon_{13}^{3}$ & 10 \\
\hline 39 & 61 & 5 & 2 & 61 & 149 & 5 \\
\hline 64 & 41 & 10 & 3 & 86 & $74=2 \cdot 37$ & 10 \\
\hline 89 & 317 & 5 & 4 & 111 & $493=17 \cdot 29$ & 5 \\
\hline 114 & $130=2 \cdot 5 \cdot 13$ & 10 & 5 & 136 & $185=5 \cdot 37$ & 10 \\
\hline 139 & 773 & 5 & 6 & 161 & $1037=17 \cdot 61$ & 5 \\
\hline 164 & 269 & 10 & 7 & 186 & $346=2 \cdot 173$ & 10 \\
\hline 189 & 1429 & 5 & 8 & 211 & $1781=13 \cdot 137$ & 5 \\
\hline 214 & $458=2 \cdot 229$ & 10 & 9 & 236 & 1129 & 10 \\
\hline 239 & $2285=5 \cdot 457$ & 5 & 10 & 261 & $109 ;$ & 25 \\
\hline 264 & $697=17 \cdot 41$ & 10 & 11 & 286 & $818=2 \cdot 409$ & 10 \\
\hline 289 & $3341=13 \cdot 257$ & 5 & 12 & 311 & $3869=53 \cdot 73$ & 5 \\
\hline 314 & $986=2 \cdot 17 \cdot 29$ & 10 & 13 & 336 & 1129 & 10 \\
\hline 339 & 4597 & 5 & 14 & 361 & $5213=13 \cdot 401$ & 5 \\
\hline 364 & $53 ;$ & 50 & 15 & 386 & $1490=2 \cdot 5 \cdot 149$ & 10 \\
\hline 389 & 6053 & 5 & 16 & 411 & $6757=29 \cdot 233$ & 5 \\
\hline 414 & $1714=2 \cdot 857$ & 10 & 17 & 436 & 1901 & 10 \\
\hline 439 & $7709=13 \cdot 593$ & 5 & 18 & 461 & 8501 & 5 \\
\hline 464 & 2153 & 10 & 19 & 486 & $2362=2 \cdot 1181$ & 10 \\
\hline 489 & $9565=5 \cdot 1913$ & 5 & 20 & 511 & $10445=5 \cdot 2089$ & 5 \\
\hline 514 & $2642=2 \cdot 1321$ & 10 & 21 & 536 & 17 & 130 \\
\hline 539 & 11621 & 5 & 22 & 561 & 12589 & 5 \\
\hline 564 & 3181 & 10 & 23 & 586 & $3434=2 \cdot 17 \cdot 101$ & 10 \\
\hline 589 & 13877 & 5 & 24 & 611 & $14933=109 \cdot 137$ & 5 \\
\hline 614 & $3770=2 \cdot 5 \cdot 13 \cdot 29$ & 10 & 25 & 636 & $4045=5 \cdot 809$ & 10 \\
\hline 639 & 16337 & 5 & 26 & 661 & 17477 & 5 \\
\hline 664 & 4409 & 10 & 27 & 686 & $4706=2 \cdot 13 \cdot 181$ & 10 \\
\hline 689 & $18989=17 \cdot 1117$ & 5 & 28 & 711 & $20221=73 \cdot 277$ & 5 \\
\hline 714 & $5098=2 \cdot 2549$ & 10 & 29 & 736 & 5417 & 10 \\
\hline
\end{tabular}


Table 3

The case of $p=13$.

$$
t=169 m-29
$$

$D=169 m^{2}-58 m+5$

$$
\begin{gathered}
t=169 m+29 \\
D=169 m^{2}+58 m+5
\end{gathered}
$$

\begin{tabular}{|c|c|c|c|c|c|c|}
\hline$t$ & $D$ & $u$ & $m$ & $t$ & $D$ & $u$ \\
\hline & & & 0 & 29 & $5 ; \varepsilon_{0}^{7}$ & 13 \\
\hline 140 & $29 ; \varepsilon_{29}^{3}$ & 26 & 1 & 198 & $58=2 \cdot 29$ & 26 \\
\hline 309 & $565=5 \cdot 113$ & 13 & 2 & 367 & 797 & 13 \\
\hline 478 & $2 ;$ & 338 & 3 & 536 & 17 ; & 130 \\
\hline 647 & $\therefore \quad \therefore$ & 13 & 4 & 705 & $2941=17 \cdot 173$ & 13 \\
\hline 816 & $985=5 \cdot 197$ & 26 & 5 & 874 & $1130=2 \cdot 5 \cdot 113$ & 26 \\
\hline 985 & 5741 & 13 & 6 & 1043 & $6437=41 \cdot 157$ & 13 \\
\hline 1154 & $1970=2 \cdot 5 \cdot 197$ & 26 & 7 & 1212 & $2173=41 \cdot 53$ & 26 \\
\hline 1323 & 10357 & 13 & 8 & 1381 & $11285=5 \cdot 37 \cdot 61$ & 13 \\
\hline 1492 & $3293=37 \cdot 89$ & 26 & 9 & 1550 & $3554=2 \cdot 1777$ & 26 \\
\hline 1661 & $653 ;$ & 65 & 10 & 1719 & $17485=5 \cdot 13 \cdot 269$ & 13 \\
\hline 1830 & $4954=2 \cdot 2477$ & 26 & 11 & 1888 & 5273 & 26 \\
\hline 1999 & $23645=5 \cdot 4729$ & 13 & 12 & 2057 & 25037 & 13 \\
\hline 2168 & $6953=17 \cdot 409$ & 26 & 13 & 2226 & $7330=2 \cdot 5 \cdot 733$ & 26 \\
\hline
\end{tabular}

Mathematical Institute

Nagoya University

The author wishes to express his hearty thanks to Professor H. Hasse who kindly pointed out that it had been forgotten to add in Lemma 4 the condition $0<a<p^{2}$, which establishes uniqueness. (27, August 1968) 